\title{
The Performance of Fine Resolution Neutron Powder Diffractometer at PTBIN-BATAN
}

\author{
A. Fajar ${ }^{*}$ and H. Mugirahardjo \\ Centre for Technology of Nuclear Industry Materials, BATAN \\ Puspiptek Serpong, Tangerang, Indonesia
}

\section{ARTICLE INFO}

Article history:

Received 15 October 2009

Received in revised form 12 April 2010

Accepted 21 April 2010

Keywords:

Neutron powder diffraction

Resolution

Crystal structure

Rietveld profile fitting

\begin{abstract}
A B S T R A C T
We describe the performance of a fine resolution neutron powder diffractometer, DN3, which has been installed at the neutron guide (NG2) in the Neutron Guide Hall about $71 \mathrm{~m}$ away from the Multipurpose G. A. Siwabessy reactor core. DN3 has a multi-detector system which consists of $32{ }^{3} \mathrm{He}$ neutron detectors and sollertype collimation. The resolution curve of the instrument was found to have little variation over a wide angular region. The best resolution is $\Delta 2 \theta$ (FWHM) of $0.23^{\circ}$ and $\Delta d / d$ of $5 \times 10^{-3}$, where $2 \boldsymbol{\theta}$ and $\boldsymbol{d}$ are scattering angle and $d$ spacing, respectively. The instrument provides sample environment of low temperatures using a cryostat having a temperature range of $10-300 \mathrm{~K}$. Several experimental results, interlaboratory comparison study and he next activities to improve the performance of DN3 equipment have also been discussed.
\end{abstract}

(C) 2010 Atom Indonesia. All rights reserved

\section{INTRODUCTION}

Powder diffraction is one of the most widely used techniques to study the structural and microstructural properties of materials. Potentially, diffraction allows determination of: long-range structure in polycrystalline materials, short-range atomic structure in disordered or amorphous materials, structural distortions, and any strain and crystal size induced changes to the structure. Accurate structure determinations are essential for understanding a material because there is a direct correlation between a materials composition, structure, and its properties.

Neutron powder diffraction, although less commonly available than x-ray diffraction due to its reliance on large-scale infrastructure, plays an essential role in these structural studies. Neutron powder diffraction has many attractive features that make it complementary to other techniques, such as $\mathrm{x}$-ray and electron diffraction. These features include the neutrons' penetrative ability, light element sensitivity, isotope dependent scattering, and its magnetic interaction. This means that the applications of neutron powder diffraction are significant and involve the fields of structural physics and chemistry, condensed-matter physics (magnetism, superconductivity), materials science, life science, earth science and engineering [1].
Neutron diffraction experiments require, however, a rather long counting time to obtain highquality data because the scattering amplitude of neutron is extremely smaller than that of X-ray. Thus, the improvement of the scattering intensity is seriously required for actual neutron diffraction experiments. On the other hand, the scattering intensity of a Bragg peak is basically in inverse proportion to the angular resolution, so that, to perform effective experiments, the intensity and resolution conditions of a neutron diffraction should be optimized according to the purpose. A detailed discussion of ideal resolution and intensity conditions for a conventional diffractometer was reported by Hewat [2].

The Neutron Spectrometry Division of Center for Technology of Nuclear Industry Material in Serpong is equipped with three neutron diffractometers: a powder diffractometer (DN1), a Four Circle Diffractometer/Texture Diffractometer (DN2) and a Fine Resolution Powder Diffractometer (DN3). The DN1 was the first instrument installed (1987) by JICA Japan and is situated in the reactor experimental hall (XHR) of the Multi-Purpose Reactor (RSG) GA Siwabessy. The two others were installed in the middle 1992 under BATANSumitomo phase III project. DN1 is now dedicated for residual stress measurement, and DN2 is for texture measurements, and DN3 is dedicated for powder measurements [3].

\footnotetext{
Corresponding author.

E-mail address: andika@batan.go.id (A. Fajar)
} 
The purpose of the present paper is to describe the details of the diffractometer and to show its performance. In the following we will describe the instrument design and list the important instrument specifications. Then we will present some typical results obtained by DN3, so as to indicate the actual performance and focus on the resolution and the line profile shapes which the latter are crucial for the quality of the Rietveld refinement. We have also made the inter-laboratory comparison between DN3 and ECHIDNA (ANSTO) instruments for measuring the apatites samples and discuss the obtained results.

\section{INSTRUMENT DETAILS OF DN3}

DN3 has been installed by BATAN at the S5 beam port of the curved neutron guide tube number two (NGT2) in the Neutron Guide Hall (NGH) about 71 meters away from the reactor core. An evacuated guide tube covers most of this distance in order to avoid attenuation of the neutron beam by air scattering. The main purpose using this curved guide is to avoid neutron with the high energy in the direct beam as well as to reduce gammas and background. Figures 1 and 2 show the instrument and the schematic diagram of DN3, respectively. The main instrumental parameters of the DN3 are described in Table 1. DN3 consists of four main units; the monochromator unit, collimators, a sample table and a multicounter system. The distances from the monochromator to the sample position, $\boldsymbol{L}_{1}$, and from the sample position to each detector, $\boldsymbol{L}_{2}$, are $2800 \mathrm{~mm}$ and $965 \mathrm{~mm}$, respectively.

Neutron flux of the white beam in NGT2 guide tube was observed to be about $10^{7} \mathrm{n} \mathrm{cm}^{-2} \mathrm{~s}^{-1}$. The white beam is monochromated by the 331 Bragg reflection of germanium single crystal. The monochromator drum has three exits corresponding to three different take-off angles, $2 \theta_{\mathrm{M}}=41.5^{\circ}, 89^{\circ}$

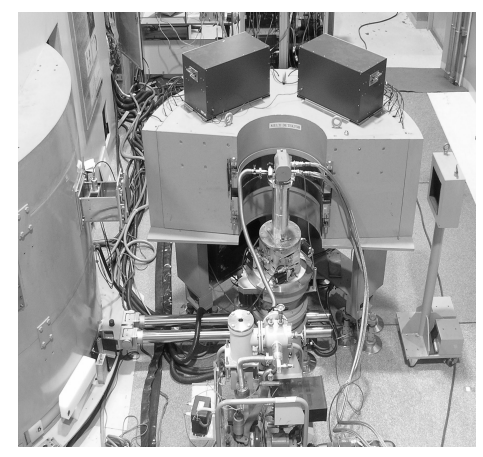

Fig. 1. The HRPD instrument. and $130^{\circ}$ which give different wavelength of monochromatic beam. At present condition the instrument is set for $2 \theta_{M}=89^{\circ}$, i.e. the wavelength of incident neutron beam, $\lambda=1.82 \AA$. The reason for this are as follows: (1) Since the flux of the white neutron beam at the wavelength range below $1 \AA$ at NGT2 guide tube is extremely small, contamination by higher-order components of reflections, such as $\lambda / 2$ reflection, can be neglected when , $\lambda=1.82 \AA$. (2) Since the neutron flux of the white beam in the guide tube is approximately constant around $2 \AA$ and the reflectivity of the monochromator is proportional to $\lambda^{3} / \sin \left(2 \theta_{M}\right)$, the flux of the monochromatic beam increases with increasing $\lambda$ around $2 \AA$. Thus, $\lambda=1.82 \AA$ gives the highest flux of the monochromatic beam in the region where the $\lambda / 2$ reflection can be neglected. (3) Resolution estimation using the formula by Caglioti et al. [4], as mentioned in detailed later, indicated that the condition of $2 \theta_{M}=89^{\circ}$ yields better resolution in the low scattering-angle region below $2 \theta=90^{\circ}$ than that yielded in the case of higher $2 \theta_{M}$. Since both crystal structure refinement and magnetic scattering measurements are important targets of DN3, higher resolution in the low scattering angle, i.e., low momentum transfer region and a rather wide $2 \boldsymbol{\theta}$ range of good resolution are required for the apparatus. From these three points of view, we use the monochromatic beam of $\lambda=1.82 \AA, 2 \theta_{M}=89^{\circ}$ on the DN3.

The Ge(331) single crystal monochromator was pressed under the pressure of $70 \mathrm{~kg} \cdot \mathrm{cm}^{-2}$ during heating at $850^{\circ} \mathrm{C}$ for one hour to increase the mosaicity of the crystal and improve the reflectivity. The dimensions of the beam are $33 \mathrm{~mm}$ width $\times 90 \mathrm{~mm}$ height at the monochromator, and $15 \mathrm{~mm}$ $\times 40 \mathrm{~mm}$ (adjustable by beam narrower) at the sample position. A monitor detector is installed in the incident monochromatic beam for normalization of the count of each measurement

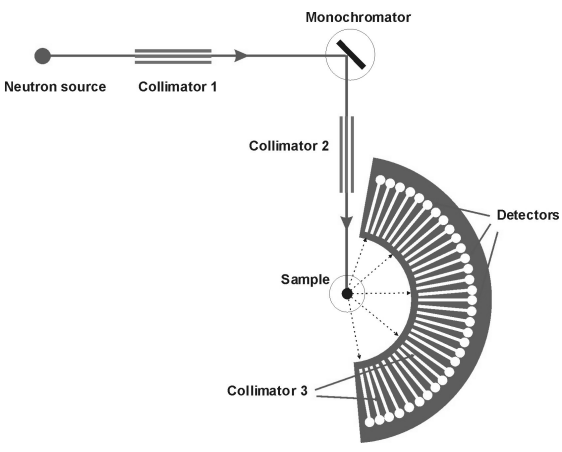

Fig. 2. Schematic diagram of HRPD. 
The detector unit consists of a detector-bank table with an air-floating system and the multidetector system in a detector shield on the detector bank table. For control of the scattering angle, DN3 has the rotation mechanism of S-axis for rotating the whole detector unit around the sample position using the air-floating system. The lowest $2 \boldsymbol{\theta}$ of the measurement range can be changed from $+10^{\circ}$ to $-170^{\circ}$. The accuracy of $2 \theta$ in an actual measurement is about $0.01^{\circ}$, and the convergence time of the angular control is just $2-3 \mathrm{~s}$ and is completely negligible in comparison with the total duration of an ordinary measurement.

Three collimators are available: before and after monochromator, and before each detector. From geometry as well as materials used for NGT it could be considered as a first collimator. The second collimator made from vertical thin mylar films coated by gadolinium oxide with angular divergences of 20' is used after the monochromator. The 32 third collimator with angular divergence of 6 , which each of them related to the one ${ }^{3} \mathrm{He}$ detector make the fine resolution measurement can be carried out. The details of the resolution using these collimation systems are described in the next section. For the accurate measurement of diffracted intensity beam, the monitor counter is set before sample to monitor the incident monochromatic beam, especially if there is a fluctuation of neutron flux from the reactor in order to get a good statistically data. The detector system consists of 32 units of ${ }^{3} \mathrm{He}$ detectors whose diameter $25 \mathrm{~mm}$. The detectors separated at equal distances by $5.0^{\circ}$ between each other which placed in the detector bank, so that DN3 can observe a powder pattern in the scattering angle of $160^{\circ}$ by only 100 step-scan repetitious, when the step angle of $2 \boldsymbol{\theta}$ is $0.05^{\circ}$ in a measurement. The counting rate of each detector is normalized by measurements of the intensity of the direct beam, incoherent scattering from vanadium, water, etc.

Since the decrease of background at the low $2 \boldsymbol{\theta}$ region which is due to air scattering is very important for magnetic scattering measurements, a $\mathrm{B}_{4} \mathrm{C}$ beam stopper is installed just after the sample position (see Fig. 1), and stops the beam transmitted through the sample. At present, when a standard sample cell and cryostat are used, the background level per detector per 1 min is about 2 counts, which is small enough in actual experiments because the incoherent scattering from a sample itself is much stronger than the background level.

A sample is positioned at the centre of the sample table as shown in Fig. 1. All the pieces of equipment are controlled by the data-acquisition program of DN3 on a PC IBM compatible computer, so that users can perform automatic measurements for extended periods of time. This considerably decreases the load on users performing the experiments. Ancillary equipments for this instrument are a furnace with maximum temperature of $900 \mathrm{~K}$, and a cryostat with closed cycle refrigerator down to $10 \mathrm{~K}$.

Table 1. Technical parameters of the fine resolution powder diffractometer DN3 at the reactor G.A. Siwabessy at the Center for Technology of Nuclear Industry Material BATAN Serpong

\begin{tabular}{|c|c|c|}
\hline Beam tube & & NG2-3 at beam port S5 (radial) $-33 \times 90 \mathrm{~mm}^{2}$ \\
\hline Take off angle & & $41.5^{\circ}, 89^{\circ}$ and $130^{\circ}$ \\
\hline Monochromator & : & $\begin{array}{l}\text { hot-pressed Ge with reflecting planes (331) normal } \\
\text { to crystal surface, mosaicity FWHM } \\
\beta=23.4^{\prime}\left(0.39^{\circ}\right), 10 \mathrm{~cm} \times 4.5 \mathrm{~cm} \times 1 \mathrm{~cm}\end{array}$ \\
\hline Wavelength & : & $1.8223 \AA$ for $\mathrm{Ge}(331)$ and $2 \theta_{\mathrm{M}}=89^{\circ}$ \\
\hline Beam narrower & & Max. $40 \mathrm{~mm}$ (horizontal) x $100 \mathrm{~mm}$ (vertical) \\
\hline Collimators & : & $\begin{array}{l}\text { 1st collimator } \alpha_{1}(\mathrm{NG} 2)=23.4^{\prime}\left(0.39^{\circ}\right) \\
\left.\text { 2nd collimator } \alpha_{2}=20^{\prime}\left(0.33^{\circ}\right) \text { (present, } 25 \times 100 \mathrm{~mm}^{2}\right), 40^{\prime} \\
\left(0.67^{\circ}\right)\left(35 \times 100 \mathrm{~mm}^{2}\right) \\
\left.\text { 3rd collimator } \alpha_{3}=6,\left(0.10^{\circ}\right) \text { (present, 20x100 } \mathrm{mm}^{2}\right), 10^{\prime} \\
\left(0.17^{\circ}\right)\end{array}$ \\
\hline Scattering angle & & $-170^{\circ}<2 \theta \mathrm{s}<+10^{\circ}$ \\
\hline Main detectors & & 32 units of ${ }^{3} \mathrm{He}$ detectors, XERAM 30NH15 \\
\hline Data acquisition & & PC IBM compatible \\
\hline
\end{tabular}


The angular resolution of the diffractometer is evaluated using observed data from Standard Reference Material (SRM) of $\mathrm{Si}, \mathrm{Cr}_{2} \mathrm{O}_{3}$, and $\mathrm{Al}_{2} \mathrm{O}_{3}$ powder samples from NBS (now NIST). A powder sample is placed in a cylindrical vanadium cell. The total 3200 data of diffraction patterns were measured over the range of $2.5-162.5^{\circ} 2 \theta$ with a step size of $0.05^{\circ}$. The full width at half maximum $(F W H M)$ and peak position, $x_{0}$, of Bragg peaks are estimated from a Gaussian fitting procedure using IGOR Pro software to an observed pattern of standard powder samples using following formalism,

$$
\begin{aligned}
& y=y_{0}+A \exp \left[-\left(\frac{x-x_{0}}{\text { width }}\right)^{2}\right] \\
& F W H M=2 \sqrt{\ln 2} \times \text { width }
\end{aligned}
$$

Next, the obtained resolution curve will be compared with the expected resolution from the conceptual design determined using Cagliotti formalism [4], as explained below. The resolution of a fixed wavelength diffractometer depends on the horizontal collimation of the neutron beams and the scattering angles at the monochromator and the sample. Since the first collimation in the guide tube, $\alpha_{1}$, and the mosaicity of the monochromator, $\beta$, are all fixed under the usual experimental conditions, the resolution of DN3 is changed by a modification of $2 \theta_{M}, \alpha_{2}$ and $\alpha_{3}$. The resolution may be described by equation derived by Cagliotti [4],

$$
[F W H M]^{2}=U \cdot \tan ^{2} \boldsymbol{\theta}_{s}+V \cdot \tan \boldsymbol{\theta}_{s}+W
$$

with the prefactors $\boldsymbol{U}, \boldsymbol{V}$ and $\boldsymbol{W}$, being complex functions of the beam divergences and of the monochromator angle $\boldsymbol{\theta}_{M}$, as following

$$
\begin{aligned}
& U=\frac{4\left(\alpha_{1}^{2} \alpha_{2}^{2}+\alpha_{1}^{2} \beta^{2}+\alpha_{2}^{2} \beta^{2}\right)}{\tan ^{2} \theta_{M}\left(\alpha_{1}^{2}+\alpha_{2}^{2}+4 \beta^{2}\right)} \\
& V=\frac{-4 \alpha_{2}^{2}\left(\alpha_{1}^{2}+2 \beta^{2}\right)}{\tan \theta_{M}\left(\alpha_{1}^{2}+\alpha_{2}^{2}+4 \beta^{2}\right)} \\
& W=\frac{\alpha_{1}^{2} \alpha_{2}^{2}+\alpha_{1}^{2} \alpha_{3}^{2}+\alpha_{2}^{2} \alpha_{3}^{2}+4 \beta^{2}\left(\alpha_{2}^{2}+\alpha_{3}^{2}\right)}{\alpha_{1}^{2}+\alpha_{2}^{2}+4 \beta^{2}}
\end{aligned}
$$

The minimum of that function occurs for

$$
\tan \theta_{s}=-\frac{V}{2 U}=\frac{4 \alpha_{2}^{2}\left(\alpha_{1}^{2}+2 \beta^{2}\right)}{8\left(\alpha_{1}^{2} \alpha_{2}^{2}+\alpha_{1}^{2} \beta^{2}+\alpha_{2}^{2} \beta^{2}\right)} \tan \theta_{M}
$$

that is, when the scattering angle at the sample is close to the monochromator angle. By using the parameter values in Table $1, \alpha_{1}=0.39^{\circ}, \alpha_{2}=0.33^{\circ}$, and $\alpha_{3}=0.10^{\circ}$, the expected resolution could be determined.

Another way to measure the resolution of two axis diffractometer is using $\Delta d / d$ expression as,

$$
\frac{\Delta d}{d}=\cot \theta \Delta \theta
$$

This equation is obtained by differentiating Bragg's equation, $2 \mathrm{~d} \sin \theta=\lambda$ where $\lambda$ is constant. In this case, $\boldsymbol{\theta}$ is the Bragg angle, i.e. half the scattering angle, and $\Delta \theta$ is FWHM of the peak in $2 \theta$.

The peak shapes are evaluated from the diffraction pattern of silicon standard sample. The curve fitting for the observed intensities was carried out by means of the Rietveld technique using RIETAN2000 [5], assuming two kinds of the pseudo-Voigt functions as the peak shape function. As is well-known, the Rietveld profile fitting analysis is an important and useful method to obtain accurate information regarding crystal structure.

Finally, the inter-laboratory compare-son of diffraction data obtained with DN3 and ECHIDNA will be presented. ECHIDNA is the name of the new neutron High Resolution Powder Diffractometer at Australia's new research reactor OPAL, ANSTO [6]. The samples used in the experiments are silicoalumino-apatites as solid oxide fuel cell electrolytes, namely $\mathrm{La}_{9.56} \mathrm{Si}_{5.5} \mathrm{~B}_{0.5} \mathrm{O}_{26}$ which measured with DN3 and $\mathrm{La}_{9.58} \mathrm{Si}_{6} \mathrm{O}_{26.38}$ with ECHIDNA. The samples and diffraction data measured with high resolution and medium resolution mode of ECHIDNA were received from the NTU, Singapore. The fitting results using Rietveld analysis will be compared to explore the performance of two equipments.

\section{RESULTS AND DISCUSSION}

\section{Resolution}

Figure 3 shows the scattering angle dependence of the resolution. The markers, open circle, square and crosses, in Fig. 3 indicate the full width at half maximum (FWHM) of Bragg peaks for an observed diffraction pattern of standard $\alpha-\mathrm{Al}_{2} \mathrm{O}_{3}$, $\mathrm{Cr}_{2} \mathrm{O}_{3}$ and silicon powder samples, respectively. The resolution is below $0.5^{\circ}$ at the scattering angle less than $120^{\circ}$. The theoretical calculation of DN3 resolution curve has been done using Eq. (2)-(3) and shown with the dashed line. At higher scattering angle the agreement between observed and calculation value were not good. This may be due to 
the horizontal divergence collimation value of long guide tube and collimator- 2 placed in the long distance between monochromator and sample table were not adequate. The curve fitting has performed using the Cagliotti equation which described by the Eq. (2) and the collimation value, $\alpha_{1}, \alpha_{2}$, and $\alpha_{3}$, as parameters.

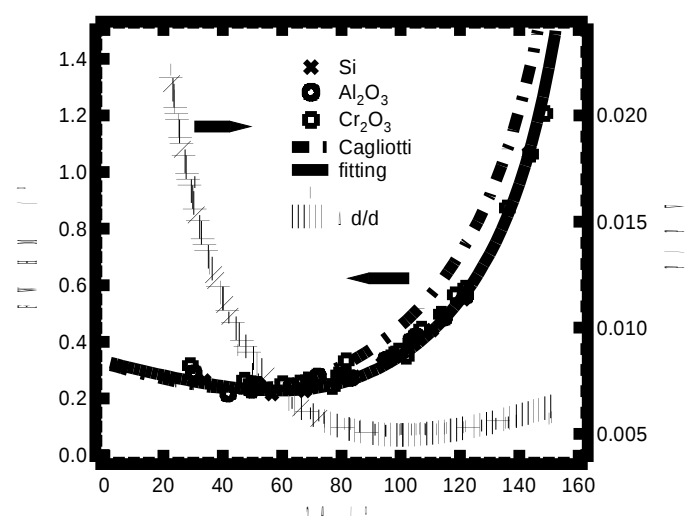

Fig. 3. Scattering angle dependence of the resolution of DN3. Open circles, square and crosses show observed FWHM of Bragg peaks of standard $\mathrm{Al}_{2} \mathrm{O}_{3}, \mathrm{Cr}_{2} \mathrm{O}_{3}$ and silicon powder samples, respectively. The solid line and dashed line are the result of the fitting and calculated value using Cagliotti formalism (left-hand scale), the dashed line (right-hand scale) is the calculated value of $\Delta d / d$ from the result of the fitting, respectively.

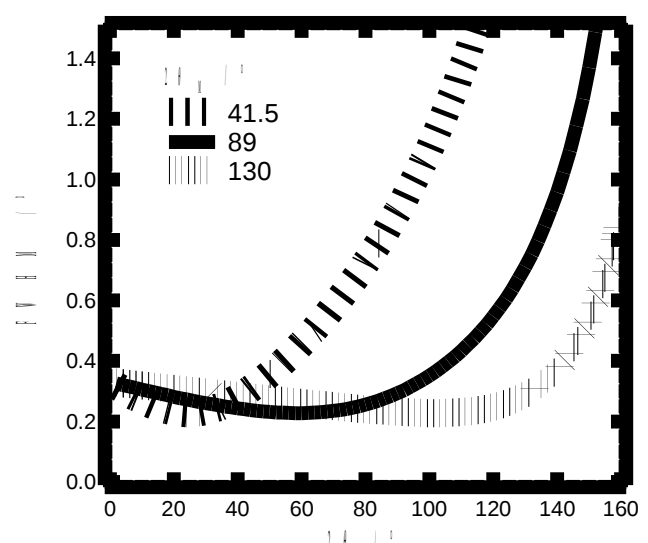

Fig. 4. Scattering angle dependence of the calculated resolution using various monchromator take-off angles with collimation values of $\alpha_{1}=0.267^{\circ}, \alpha_{2}=0.325^{\circ}$ and $\alpha_{3}=0.136^{\circ}$.

The solid line in Fig. 3 indicates the result of the fitting. The coincidence of the calculation and the observed FWHM is excellent in the whole $2 \theta$ range of measurement. The parameters of the resolution function, $\alpha_{1}, \alpha_{2}$ and $\alpha_{3}$ are obtained as $\alpha_{1}=0.267(8)^{\circ}, \alpha_{2}=0.325(2)^{\circ}$, and $\alpha_{3}=0.136(1)^{\circ}$, respectively. The $\alpha_{1}$ value is obtained smaller than given value at Table 1 . Another dashed line in Fig. 3 shows the scattering angle dependence of $\Delta d / d$ (right hand axis), where $d$ is the $d$-spacing of the reflections, which was calculated using Eq. (5). From Fig. 3, the best resolution is $13.6^{\prime}\left(0.23^{\circ}\right)$ of
$\Delta(2 \theta) / F W H M$ and $5 \times 10^{-3}$ of $\Delta d / d$. This result implies that the changes in $d$ - spacings can be measured down to the $10^{-4}$ level. Since these results are quite better than the expected resolution from the conceptual design, DN3 has satisfactory resolution in the whole experimental $2 \theta$ range.

Figure 4 also shows the scattering angle dependence of calculated resolution using various kinds of monochromator take-off angles and collimation values of $\alpha_{1}=0.267^{\circ}, \alpha_{2}=0.325^{\circ}$, and $\alpha_{3}$ $=0.136^{\circ}$. The high resolution mode varies until higher scattering angle as increasing the take-off angle. The values of prefactors $U, V$ and $W$ for various take-off angles are described in Table 2.

Table 2. The calculated prefactors $\mathrm{U}, \mathrm{V}$ and $\mathrm{W}$ of Cagliotti equation for various take-off angle with $\alpha_{1}=0.267^{\circ}, \alpha_{2}=0.325^{\circ}$, and $\alpha_{3}=0.136^{\circ}$

\begin{tabular}{|l|l|l|l|}
\hline & $2 \theta_{\mathrm{M}}=89^{\circ}$ & $2 \theta_{\mathrm{M}}=41.5^{\circ}$ & $2 \theta_{\mathrm{M}}=130^{\circ}$ \\
\hline $\mathrm{U}$ & 0.182 & 1.222 & 0.038 \\
\hline $\mathrm{V}$ & -0.205 & -0.532 & -0.094 \\
\hline $\mathrm{W}$ & 0.110 & 0.110 & 0.110 \\
\hline
\end{tabular}

Broadly, the measurement of powder diffraction pattern can be separated into the experiment that primarily need intensity and the experiment that primarily need resolution. Fig. 5 shows the qualitative relationship between experiment, resolution, and intensity; the trend from lower-left to upper-right reflects the limiting nature of a powder diffractometer (the Intensity-Resolution trade-off) [1].

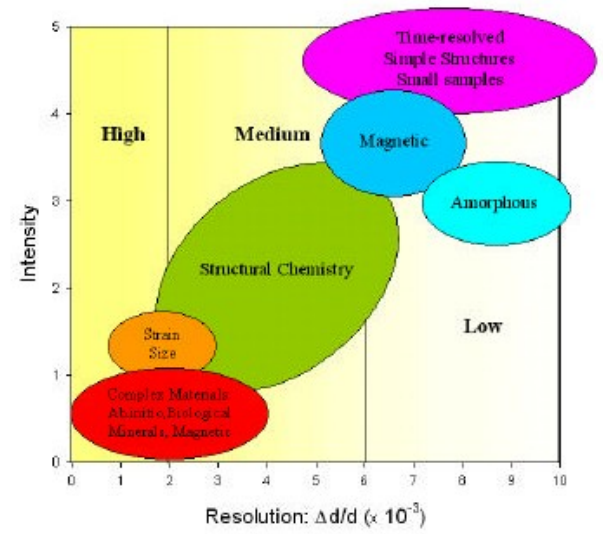

Fig. 5. Guide to the mind's eye of the relation between experiment, intensity, and resolution [1].

It is possible with high-resolution diffractometers like D2B diffractometer located at Institute Laue-Langevin (ILL) in France to attain resolutions FWHM of $0.1^{\circ}$ equivalent to $\Delta d / d \approx 5 \times 10^{-4}$ [7]. This is in the order of the limit set by the line broadening due to the primary extinction in the crystallites. Opening up the beam divergence and going to larger $d$-spacings for the 
monochromator (that is, lower take-off angles for a given wavelength) increases flux at the expense of resolution. This can be important in the case of kinetic studies. From Fig. 5, DN3 comparing with the recent developed neutron powder diffractometer can be classified into medium resolution powder diffractometer.

\section{Crystal structure refinement}

Examples of crystal structure refinement on DN3 are given below. Fig. 6 represents a typical pattern of powder diffraction obtained using DN3. The sample is standard powder sample silicon (NBS 640a: $a=5.43094 \AA$ ) which has the diamond structure (space group: $F d \overline{3} m$, A-227) placed in a standard vanadium cylindrical cell. In the figure, a result of the Rietveld profile fitting analysis by means of RIETAN2000, and the difference between observation and calculation results are given. The usual refinement factors are determined as follows; $R_{\mathrm{wp}}=16.86, \quad R_{\mathrm{e}}=14.69, \quad \mathrm{~s}=R_{\mathrm{wp}} /$ $R_{\mathrm{e}}=1.1481, R_{\mathrm{I}}=5.65$ and $R_{\mathrm{F}}=3.38$. From the fitting procedure, the lattice constant, $a$, and isotropic atomic displacement parameter of silicon, $B$, are determined to be 5.4305(4) $\AA$ and 0.55(7) $\AA^{2}$, respectively, when $\lambda=1.8223 \AA$. The obtained value corresponds to that of the standard sample determined in NBS.

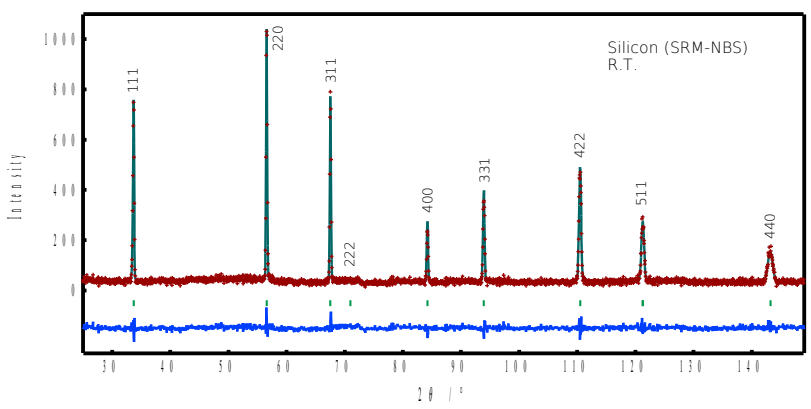

Fig. 6. Rietveld refinement using neutron diffraction data for compound Si powder. The points are the data and the line through the points is the result from the refinement. The Bragg peak positions are shown as short vertical lines. At the bottom is shown the difference between the data and the calculated profile.

\section{Peak shapes}

The observed Si powder diffraction pattern was used for the evaluation of peak shapes and peak shape function using in Rietveld analysis. Crosses in Fig. 7 represent observed Bragg peak shapes of a standard silicon powder sample. The solid and dashed lines are results of curve fitting and the difference between the observation and calculation results, respectively. The calculated peak shapes reproduce the observed Bragg peaks naturally. Two typical set of peak shape function parameters is shown in Table 3. These data were useful as the initial value within input file data when performing the Rietveld analysis. The peak shape of 220 and 331 Bragg peak has a slight tail at the low scattering angle side of the peak. The asymmetry is also observed in Bragg peaks at the lower scattering angle regions, and shows a tendency to increase with decreasing scattering angle. The umbrella effect which is due to the scattering angle dependence of divergence of a Debye-Sherrer cone causes the distortion of the peak shape at the low scattering angle region, because simulation calculations including the umbrella effect represent the distortion of Bragg peaks very well [8].
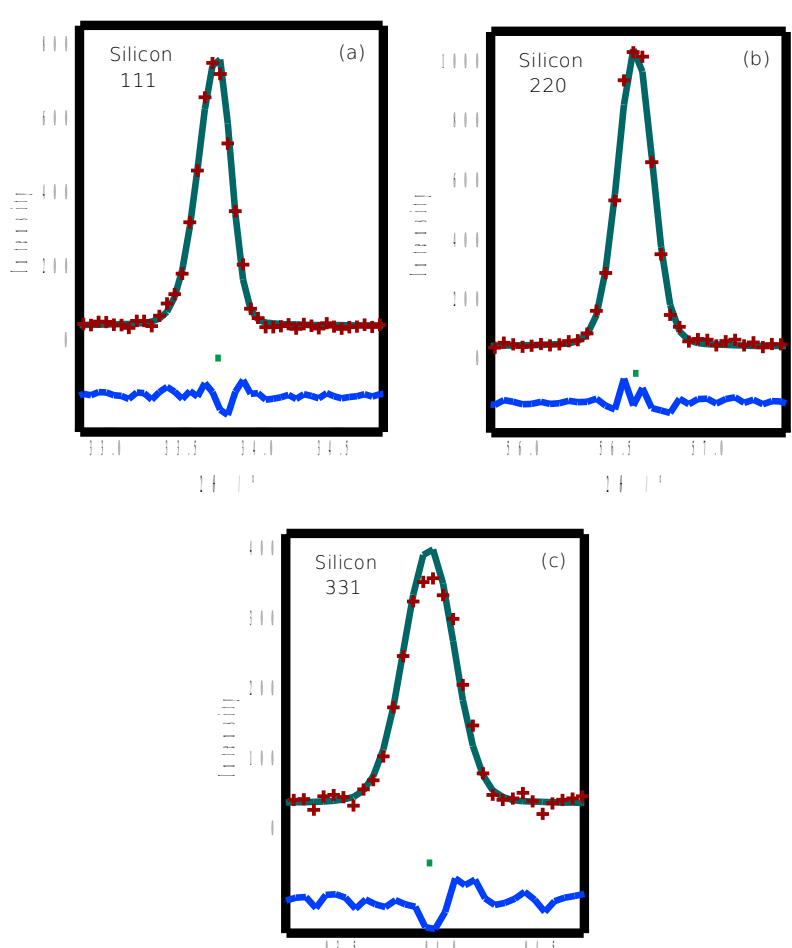

Fig. 7. Peak shape of (a) 111, (b) 220 and (c) 331 Bragg peaks of a standard silicon powder sample. Crosses, solid line and dashed lines indicate the observed Bragg intensities, fitted profile curves calculated using RIETAN2000 and the difference between the calculation and observation results.

From the results, we can say that narrow and well-described Bragg peak, suitable for high-quality crystal structure refinement, are observed by DN3, although there is still some room for improvement in the peak profile function. A typical result of crystal structure refinement using the Rietveld technique is given in the following section.

Table 3. Two typical set of profile peak shape function parameters of DN3 for silicon powder samples. 


\begin{tabular}{|l}
\hline \# TCH's pseudo-Voigt function made asymmetric by Howard's \\
method. \\
\# FWHM parameters of the Gauss function, U, V, W, and P. \\
GAUSS01 2.22188E-2 -1.96127E-2 1.03769E-2 0.0 1110 \\
\# FWHM parameters of the Lorentz function, X, Xe, Y, and Ye. \\
LORENTZ01 1.40593E-2 0.0 1.86585E-2 0.0 1010 \\
\# Asymmetry parameter, As, plus five dummies. \\
ASYM01 0.162166 0.0 0.0 0.0 0.0 0.0 100000 \\
\hline \# Non-relaxed reflections: split pseudo-Voigt function \\
\# Relaxed reflections: Modified split pseudo-Voigt function. \\
\# FWHM parameters, U, V, and W, plus a dummy. \\
FWHM12 0.171334 -2.05327E-1 0.110265 0.0 1110 \\
\# Asymmetry parameters, a0, a1, and a2 plus a dummy. \\
ASYM12 1.03723 0.545391 -1.62445E-1 0.0 1110 \\
\# Decay parameters, eta_L0, eta_L1, eta_H0, and eta_H1. \\
ETA12 0.14815 8.13176E-3 0.171053 -1.07801E-2 1111 \\
\# Asymmetric-broadening parameters, Ue and Pe. \\
ANISOBR12 9.46397E-6 0.0 10
\end{tabular}

\section{Inter-laboratory comparison}

As mentioned in previous paper, the present intensities and resolution of DN3 are comparable to those obtained from High Resolution Powder Diffractometer at ANSTO before they upgraded their neutron flux [9]. Here the comparison the diffraction pattern measured with DN3 and a of new HRPD (ECHIDNA) at ANSTO will be presented.

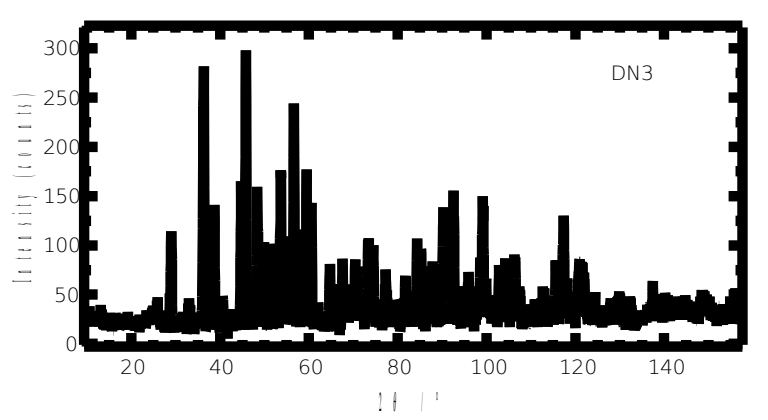

Fig. 8. Diffraction pattern for $\mathrm{La}_{9.56} \mathrm{Si}_{5.5} \mathrm{~B}_{0.5} \mathrm{O}_{26}$ data from DN3.

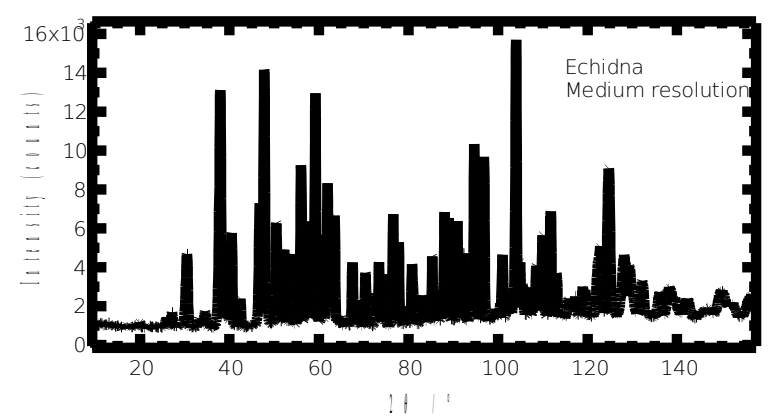

Fig. 9. Diffraction pattern for $\mathrm{La}_{9.58} \mathrm{Si}_{6} \mathrm{O}_{26.38}$ data from ECHIDNA (medium resolution mode).

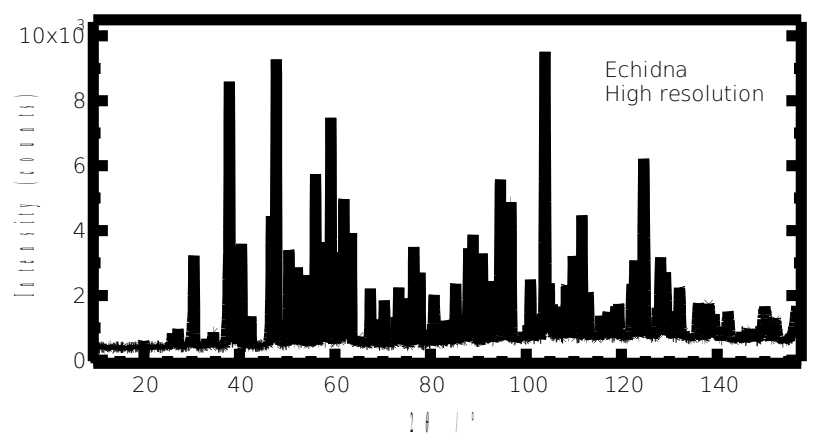

Fig. 10. Diffraction pattern for $\mathrm{La}_{9.58} \mathrm{Si}_{6} \mathrm{O}_{26.38}$ data from ECHIDNA (high resolution mode).

Figures 8 shows the diffraction pattern of $\mathrm{La}_{9.56} \mathrm{Si}_{5.5} \mathrm{~B}_{0.5} \mathrm{O}_{26}$ obtained by DN3, while Fig. 9 and Fig. 10 represent two data set that collected using ECHIDNA from the same sample of the apatite phase $\mathrm{La}_{9.58} \mathrm{Si}_{6} \mathrm{O}_{26.38}$, respectively. The first pattern (Fig. 9) was obtained in routine 'medium resolution' mode for 2 hours, while second pattern in 'highresolution' mode for 12 hours measurement.

The absolute counts or counts/time for the strongest peak around $2 \theta=45.8^{\circ}$ for DN3 and $2 \theta=47.7^{\circ}$ for ECHIDNA in sets of data were resumed in Table 4. Clearly, ANSTO are getting higher counts because they have a higher neutron flux. 


\begin{tabular}{|l|c|c|c|}
\hline & Intensity (counts) & Time (hour) & Intensity/Time (counts/hour) \\
\hline DN3 & 298 & 39 & $7.6(0.0021 \mathrm{c} / \mathrm{s})$ \\
\hline ECHIDNA (high res. mode) & 9277 & 12 & $773(0.2148 \mathrm{c} / \mathrm{s})$ \\
\hline ECHIDNA (medium res. mode) & 14173 & 2 & $7087(1.9685 \mathrm{c} / \mathrm{s})$ \\
\hline
\end{tabular}

Regarding their high intensities, from the Rietveld analysis using TOPAS, it does not really make that much difference in the analysis and the refined structures from each facilities data are very comparable, as shown in Table 5 [10]. The only real difference comes up the values obtained for the $R_{\mathrm{wp}}$ reliability factor and from the BATAN data these are generally higher but this just reflects the differences in signal to background ratios. The formula of $R_{\mathrm{wp}}$ is as follows [11]:

$$
R_{w p}=\left\{\frac{\sum_{i} w_{i}\left[y_{i}(o)-y_{i}(c)\right]^{2}}{\sum_{i} w_{i}\left[y_{i}(o)\right]^{2}}\right\}
$$

It is clear that if the background is higher, the $R_{\mathrm{wp}}$ value will be smaller. The $\mathrm{R}_{\text {Bragg }}\left(R_{\mathrm{b}}\right)$ reliability factors which reflect the structural model used are virtually identical from both ANSTO and BATAN data sets.

Table 5. Comparison of reliability factors from Rietveld analysis results of Echidna ANSTO (high resolution mode) and BATAN neutron diffraction data [10]

\begin{tabular}{|l|c|c|}
\hline Composition $\boldsymbol{x}$ & $\mathbf{L a}_{9.58} \mathbf{S i}_{6} \mathbf{O}_{\mathbf{2 6 . 3 8}}$ & $\mathbf{L a}_{9.56} \mathbf{S i}_{5.5} \mathbf{B}_{\mathbf{0 . 5}} \mathbf{O}_{\mathbf{2 6}}$ \\
\hline Space Group & $P 6_{3} / m$ & $P 6_{3} / m$ \\
\hline$a(\AA)$ & $9.7157(1)$ & $9.6781(5)$ \\
\hline$c(\AA)$ & $7.1831(1)$ & $7.1940(4)$ \\
\hline$V\left(\AA^{3}\right)$ & $587.20(1)$ & $583.55(6)$ \\
\hline GOF & 2.44 & 1.12 \\
\hline$R_{\exp }(\%)$ & 3.24 & 16.42 \\
\hline$R_{w p}(\%)$ & 7.91 & 18.32 \\
\hline$R_{p}(\%)$ & 6.08 & 14.03 \\
\hline$R_{b}(\%)$ & 3.04 & 3.62 \\
\hline
\end{tabular}

The main difference is the precision of the two instruments [10]. The TOPAS output for ANSTO data gives atomic positions precise to the 4th decimal place for oxygen atoms whereas the BATAN is precise to the 3rd decimal place. This obviously is also reflected in the refined bond lengths where the ANSTO and BATAN are precise to the 3rd and 2nd decimal places respectively. They are however in good agreement with each other within their own errors and are good enough for analysis.
These results will be discussed in order to improve the performance of DN3. From Fig. 10, it can be seen that ECHIDNA data have higher peak to background ratio and the high resolution varies until high scattering angle. It can be realized in DN3 by rotating the take-off angle of monochromator to $130^{\circ}$ as calculated in Fig. 4, but the obtained intensity and wavelength have to be considered. In order to increase the intensity, ECHIDNA is using the asymmetric configuration and vertical focusing of hot-pressed Ge monochromator [6]. While DN3 is only using one slab hot-pressed Ge monochromator. For the present DN3, it is possible to perform asymmetric monochromator but for vertical focusing, more budget will be needed.

\section{CONCLUSIONS}

The experimental results mentioned above indicate that DN3 exhibits satisfactory performance in term of resolution though fairly poor neutron flux in sample position. The best resolution is FWHM of $0.23^{\circ}$ (13.6') and $\Delta d / d \approx 5 \times 10^{-4}$, and measurement Q-range is from $0.3 \AA^{-1}$ to $6.7 \AA^{-1}$. For better performance of $\mathrm{DN} 3$, improvement of the equipment is still needed in particular the neutron flux at sample position.

\section{ACKNOWLEDGEMENTS}

The authors would like to extends our thank to Dr. Tom Baikie from Nanyang Tecnology University, Singapore, for providing us the apatites samples, neutron data from ECHIDNA ANSTO, and discussion about the performance of DN3.

\section{REFERENCES}

1. Anonymous, Report on Neutron Powder Diffraction for the Australian Replacement Research Reactor, Neutron Powder Diffraction Workshop, ANSTO, October 17 - 18, (2000). (http://www.ansto.gov.au/data/assets/pdf file/00 17/3563/w1report.pdf).

2. A.W. Hewat, Nucl., Instrum. \& Methods $\mathbf{1 2 7}$ (1975) 361. 
3. A. Fajar, T.H. Priyanto, E. Santoso, H. Mugirahardjo, N. Suparno, and A. Purwanto, Neutron News 18 (2007) 13.

4. G. Cagliotti, A. Paoletti, and F.P. Ricci, Nucl. Instrum. \& Methods 3 (1958) 223.

5. F. Izumi and T. Ikeda, Mater. Sci. Forum 198 (2000) 321.

6. K.D. Liss, B. Hunter, M. Hagen, T. Noakes, S. Kennedy, Physica B 385-386 (2006) 1010.

7. A.W. Hewat, Mater. Sci. Forum 9 (1986) 69.
8. K. Ohoyama, T. Kanouchi, K. Nemoto, M. Ohashi, T. Kajitani, and Y. Yamaguchi, Jap. J. Appl. Phys. 37 (1998) 3319.

9. A. Fajar, H. Mugirahardjo, Bharoto, E. Santoso, Ind. J. Mat. Sci., December Special Edition 27 (2008) 1.

10. T. Baikie, Private Communication.

11. R.A. Young, E. Prince, R.A. Sparks, J. Appl. Crystallogr. 15 (1982) 357. 
